\title{
Brucella induces unfolded protein response and inflammatory response via GntR in alveolar macrophages
}

\author{
Dong Zhou ${ }^{1,2, *}$, Fei-Jie Zhi ${ }^{1,2, *}$, Mao-Zhen Qi, ${ }^{1,2}$, Fu-Rong Bai ${ }^{1,2}$, Guangdong Zhang ${ }^{1,2}$, \\ Jun-Mei Li ${ }^{1,2}$, Huan Liư ${ }^{1,2}$, Hua-Tao Chen ${ }^{1,2}$, Peng-Fei Lin ${ }^{1,2}$, Ke-Qiong Tang ${ }^{1,2}$, Wei \\ Liu ${ }^{1,2}$, Ya-Ping Jin ${ }^{1,2}$ and Ai-Hua Wang ${ }^{2}$ \\ ${ }^{1}$ Key Laboratory of Animal Biotechnology of The Ministry of Agriculture, Northwest A\&F University, Yangling, China \\ ${ }^{2}$ College of Veterinary Medicine, Northwest A\&F University, Yangling, China \\ *These authors have contributed equally to this work \\ Correspondence to: Ya-Ping Jin, email: yapingjin@163.com \\ Ai-Hua Wang, email: aihuawang@126.com \\ Keywords: Brucella. suis S2; goat alveolar macrophages; unfolded protein response; cytokines \\ Received: October 03, $2017 \quad$ Accepted: December 04, $2017 \quad$ Published: December 26, 2017 \\ Copyright: Zhou et al. This is an open-access article distributed under the terms of the Creative Commons Attribution License 3.0 \\ (CC BY 3.0), which permits unrestricted use, distribution, and reproduction in any medium, provided the original author and source \\ are credited.
}

\section{ABSTRACT}

Brucella is an intracellular bacterium that causes the zoonosis brucellosis worldwide. Alveolar macrophages (AM) constitute the main cell target of inhaled Brucella. Brucella thwarts immune surveillance and evokes endoplasmic reticulum (ER) stress to replicate in macrophages via virulence factors. The GntR regulators family was concentrated as an important virulence factor in controlling virulence and intracellular survival of Brucella. However, the detailed underlying mechanism for the host-pathogen interaction is poorly understood. In this study the BSS2_II0438 mutant ( $\Delta$ GntR) was constructed. The type IV secretion system (T4SS) virulence factor genes (VirB2, VirB6, and VirB8) were down-expression in $\Delta$ GntR. $\Delta$ GntR could infect and proliferate to high titers in GAMs without a significant difference compared with the parental strain. $\Delta G$ GntR infection increased the expression of ER stress marker genes GRP78, ATF6, and PERK in the early stages of its intracellular cycle but decreased the expression of these genes in the late stages. $\Delta G$ GtR increased greatly the number of Brucella CFUs in the inactive ER stress state in GAMs. Meanwhile, $\Delta$ GntR infection increased the levels of IFN- $Y, I L-1 \beta$, and TNF- $\alpha$, indicating $\Delta$ GntR could induce the secretion of inflammatory but not anti-inflammatory cytokines IL-10. Taken together, our results clarified the role of the GntR in B. suis. $\mathbf{S 2}$ virulence expression and elucidated that GntR is potentially involved in the signaling pathway of the Brucellainduced UPR and inflammatory response in GAMs.

\section{INTRODUCTION}

Brucellosis, caused by the genus Brucella, is one of the most prevalent zoonotic diseases worldwide with more than 500,000 new people infections annually [1-3]. Brucellosis can be transmitted to human and domestic animals via direct contact with animals, consumption of contaminated dairy products, and inhalation of infected aerosols, etc, among which airborne transmission have been reported to be the most frequent in abattoirs and rural areas [4]. Following inhalation, Brucella infects alveolar macrophages (AM) and resides within Brucella containing vacuole $(\mathrm{BCV})$ that constitute the intracellular replicative niche $[5,6]$. The special replication strategy encourages the bacteria to evade immune surveillance, inhibit host cell apoptosis, complicate vaccine development, which cause a significant economic and health burden [7]. In China, the live attenuated strain Brucella. suis. Vaccine strain 2 (B. suis. S2) is most widely used vaccine for prevention and control of brucellosis in sheep and other domestic animals [8]. 
Alveolar macrophages perform different protective functions from other phagocyte during infection. The TNF- $\alpha$ response is different in human or murine AM with monocytes induced by Mycobacterium tuberculosis or heat-killed Staphylococcus aureus $[9,10]$. AM also differ from other monocytic/macrophagic populations in susceptibility to infection or the intracellular replication kinetics for virus $[11,12]$. Interestingly, the anti-inflammatory cytokine IL-10 response is lack by murine AM comparison with peritoneal macro upon lipopolysaccharide exposure [13]. Notably, a recent study showed that $B$. abortus invades and replicates in murine AM and induces significantly increase of TNF- $\alpha$, IL- $1 \beta$, IL-6 and IL-12 in AM from different germline mice [4]. However, the interaction of B. suis. S2 with the AM has scarcely been studied.

Brucella resides within BCVs after phagocytes uptake to avoid degradation within the lysosomal compartments. The BCV undergo maturation and became endosomal BCV (eBCV) with a marker LAMP-1 during the first $8 \mathrm{hr}$ postinfection (pi) [14]. In macrophages, $\sim 10 \%$ BCVs avoid end-stage lysosomes and fuse with the Endoplasmic reticulum (ER) membranes [5]. ER, a large membrane-bound organelle, governs cellular homeostasis by controlling the processing and folding of proteins [15]. During ER fusion, Brucella dramatically restructures the ER and disrupts ER homeostasis, inducing ER stress and triggering the unfolded protein response (UPR). In mammals, the UPR is signaled through three sensors that are in the ER membrane: inositol-requiring kinase 1 (IRE1), pancreatic ER eIF2a kinase (PERK), and activating transcription factor 6 (ATF6). In response to ER stress, the binding immunoglobulin protein (GRP78) is recruited away from PERK, IRE1, and ATF6 to promote protein folding capacity. The activation of these ER stress sensors enhances the transcription of chaperones and transcription factor C/EBP-homologous protein (CHOP), which are involved in apoptosis to restore cellular homeostasis. Many studies have shown that the induction of the UPR following Brucella infection promotes the intracellular growth of these bacteria [16, 17]. The virulence factors $\mathrm{TcpB}$ and $\mathrm{VceC}$ induced the UPR in macrophages, supporting Brucella intracellular replication [18]. Most recently, the GntR family of regulators was considered as virulence factors that plays a critical role in controlling virulence and the intracellular survival of B. abortus [19-21]. Several members of gntR family contribute to pathogenesis of brucellosis [19]. However, the relevance of UPR and GntR virulence in goat alveolar macrophages has not yet been elucidated.

In this study, to test whether gntR has any influence on B. suis. S2 virulence, a gntR (BSS2_II0438) mutant $\Delta \mathrm{GntR}$ was constructed and its virulence was evaluated in dairy goat alveolar macrophages (GAMs). Our results showed that three T4SS structural proteins (virB 2, 6, 8) are down-expressed in the GntR mutant. $\Delta \mathrm{GntR}$ activated UPR as evident by the increase in GRP78 and CHOP expression. Changes in the UPR influenced the proliferation of $B$. suis. S2 and $\Delta$ GntR in GAMs. 4 Phenyl butyric acid (4PBA), a pharmacologic chaperone that inhibits the UPR, substantially increasing replication and even higher in $\Delta \mathrm{GntR}$. Furthermore, $\Delta \mathrm{GntR}$ induced the secretion of the inflammatory cytokines IFN- $\gamma$, TNF- $\alpha$ and IL- $1 \beta$ but not anti-inflammatory cytokine IL-10. These results may reveal a possible mechanism for GntR to be used to decrease Brucella virulence and provide information for the further investigation of Brucella novel vaccine development.

\section{RESULTS}

\section{Construction of $\Delta \mathrm{GntR}$}

The GntR gene deletion mutant ( $\Delta \mathrm{GntR})$ based on B. suis. S2 was successfully construction. $\Delta \mathrm{GntR}$ was verified by PCR amplification (Figure 1B and 1C). The results showed that the GntR gene was not amplified in the strain $\Delta \mathrm{GntR}$. However, the GntR gene was amplified in the strain B. suis. S2. Next, qRT-PCR was performed to quantify the expression of GntR. The results indicated GntR was not transcribed in $\Delta \mathrm{GntR}$ (date not shown). In addition, the lack of expression of GntR in $\Delta \mathrm{GntR}$ was verified by Western blotting (Figure 1D).

\section{GntR regulates the expression of T4SS}

To investigate the regulatory role of GntR on the T4SS, 6 genes related to T4SS were chosen for the qRTPCR assay. As shown in Figure 2, compared with B. suis. $\mathrm{S} 2$, three genes (vir B2, 6, 8) were decreased, and three genes (vir B1, 9, 11) showed no significant difference. These results deduces that T4SS-related genes are among the most GntR-regulated genes.

\section{$\Delta$ GntR infects and replicates in GAMs}

GAMs were chosen (Supplementary Figure 1) and infected with $B$. suis. $\mathrm{S} 2$ or $\Delta \mathrm{GntR}$ at $100 \mathrm{MOI}$ to evaluate whether the GntR gene was involved in bacterial intracellular survival and adherence. The bacterial adherence capacity of $\Delta \mathrm{GntR}$ was not significantly different (data not shown), and the bacterial intracellular survival of $\Delta \mathrm{GntR}$ was also not significantly different (Figure 3 ). To determine whether the $\Delta \mathrm{GntR}$ strain could efficiently exclude $\mathrm{eBCV}$ for intracellular trafficking within AM cells, we measured the number of LAMP-1-positive eBCV at 0 and $24 \mathrm{~h}$ hpi. The results showed that the number of LAMP-1-positive eBCV was not significantly different (Figure 4). These results were consistent with the bacterial intracellular survival.

\section{The $\Delta$ GntR mutant triggers ER stress}

To investigate whether GntR was involved in ER stress, GAMs were infected with B. suis. S2 or $\Delta \mathrm{GntR}$, 
and the ER stress marker molecules (GRP78 and CHOP) were analyzed by qRT-PCR. The mRNA expression of GRP78 was increased after $\Delta \mathrm{GntR}$ mutant infection at 0 , 6 and $12 \mathrm{~h}$ post-infection and was decreased at 24 and 48 h compared with the uninfected and B. suis. S2 groups (Figure 5A).

Western blotting results showed that the expression of GRP78 was more strongly in the B. suis. S2 and $\Delta \mathrm{GntR}$ mutant infection groups than in the uninfected group at 0,12 , and $24 \mathrm{~h}$ post infection. In line with the mRNA expression, GRP78 protein expression was enhanced in the $\Delta$ GntR mutant infection group and B. suis. S2 group compared with that in control group at 0,12 and $24 \mathrm{~h}$ (Figure 5C, 5D). CHOP mRNA expression in the $\Delta \mathrm{GntR}$ group was elevated compared with that in the uninfected group at 0 and $48 \mathrm{~h}$ (Figure 5B). These results showed that GntR is involved directly or indirectly in ERS and triggered UPR.

To further investigate the UPR pathway during Brucella infection, the activation of three UPR sensors was detected by qRT-PCR after infection with $B$. suis. $\mathrm{S} 2$ or $\triangle \mathrm{GntR}$. The expression of PERK mRNA was increased in both the B. suis. S2- and $\Delta \mathrm{GntR}$-infected groups compared with that in the uninfected group at 0

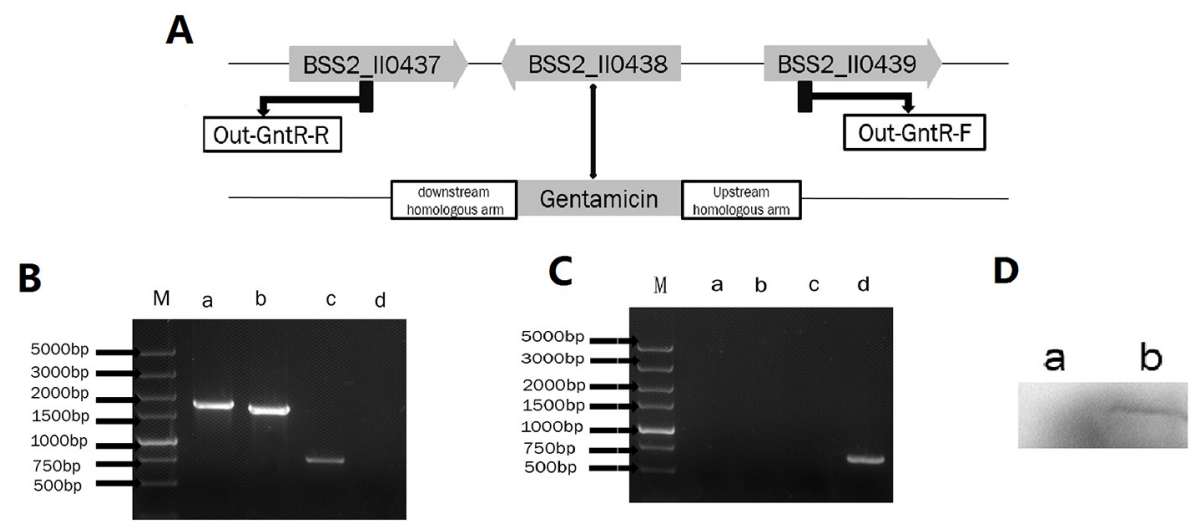

Figure 1: Identification of the $\Delta$ GntR strain. (A) Schematic of the construction of the $\Delta$ GntR strain. (B) Identification of the GntR gene deletion by PCR amplification in the $\Delta$ GntR strain. Lane M: DNA marker DL5000. Lane a: combination of the GntR upstream gene and gentamicin gene. Lane b: combination of the GntR downstream gene and gentamicin gene. Lane c: gentamicin gene. Lane d: GntR gene. (C) Identification of the GntR gene by PCR amplification in the B. suis strain. S2. Lane a: combination of the GntR upstream gene and gentamicin gene. Lane b: combination of the GntR downstream gene and gentamicin gene. Lane c: gentamicin gene. Lane d: GntR gene. (D) Western blotting confirmed the expression of the GntR gene in the $\Delta$ GntR strain. Lane a: $\Delta$ GntR strain; Lane b: B. suis. S2 strain (positive control).

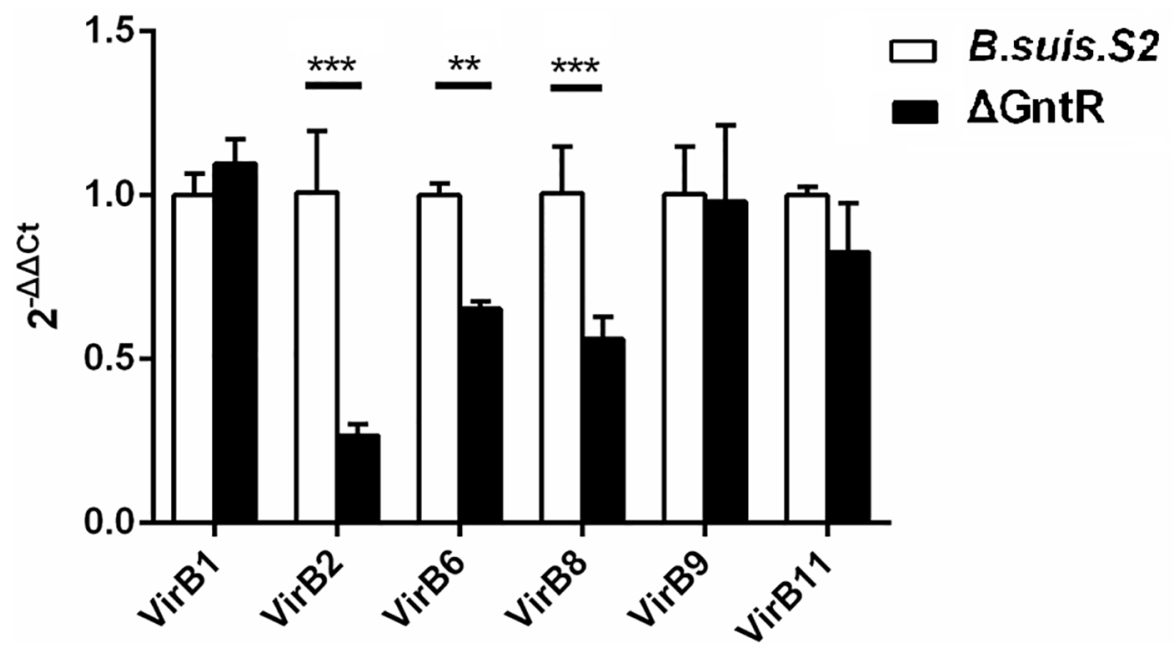

Figure 2: Transcriptional level of T4SS genes obtained by qRT-PCR in the $\boldsymbol{B}$. suis. S2 and $\Delta$ GntR strains. Strains $B$. suis. $\mathrm{S} 2$ and $\Delta \mathrm{GntR}$ were collected at the exponential phase and resuspended in Trizol for RNA purification. VirB1, VirB2, VirB6, VirB8, VirB9, VirB11 gene expression was detected by quantitative real-time PCR with normalization to 16sRNA. Data are presented as the means \pm standard error for triplicate infection samples. ${ }^{* *} P<0.01,{ }^{* * *} P<0.001$. 


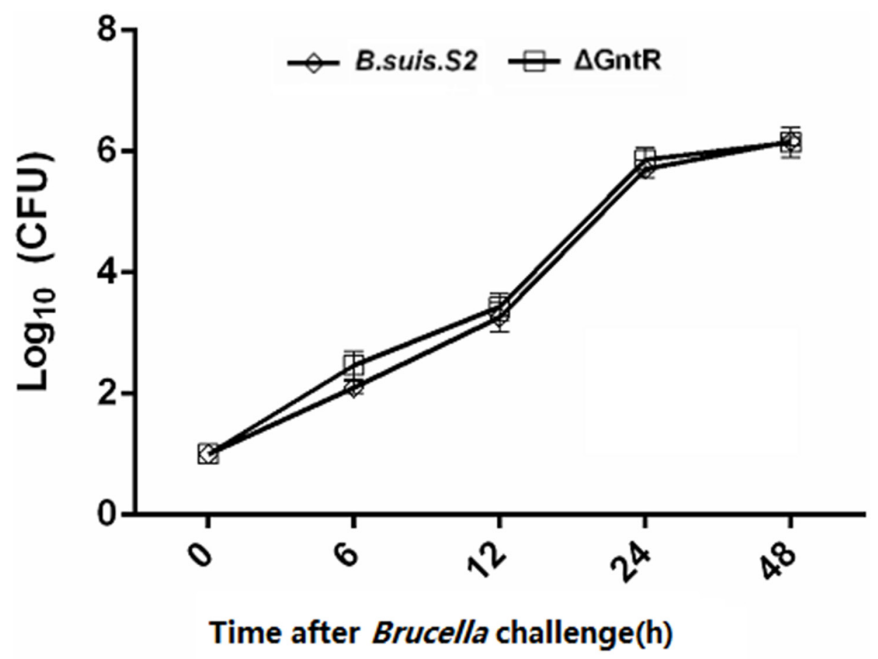

Figure 3: Intracellular survival within GAMs of the B. suis. S2 and $\mathbf{\Delta G n t R}$ strains. The GAMs were infected with wild type (B. suis. S2, rhombus) or GntR mutant Brucella ( $\Delta \mathrm{GntR}$, square). At different times following infection, cell was lysed and CFU determined by transfer to dilution plates. Error bars depict standard deviations of quadruplicate determinations. The data are shown as the means \pm standard error from 3 independent experiments.

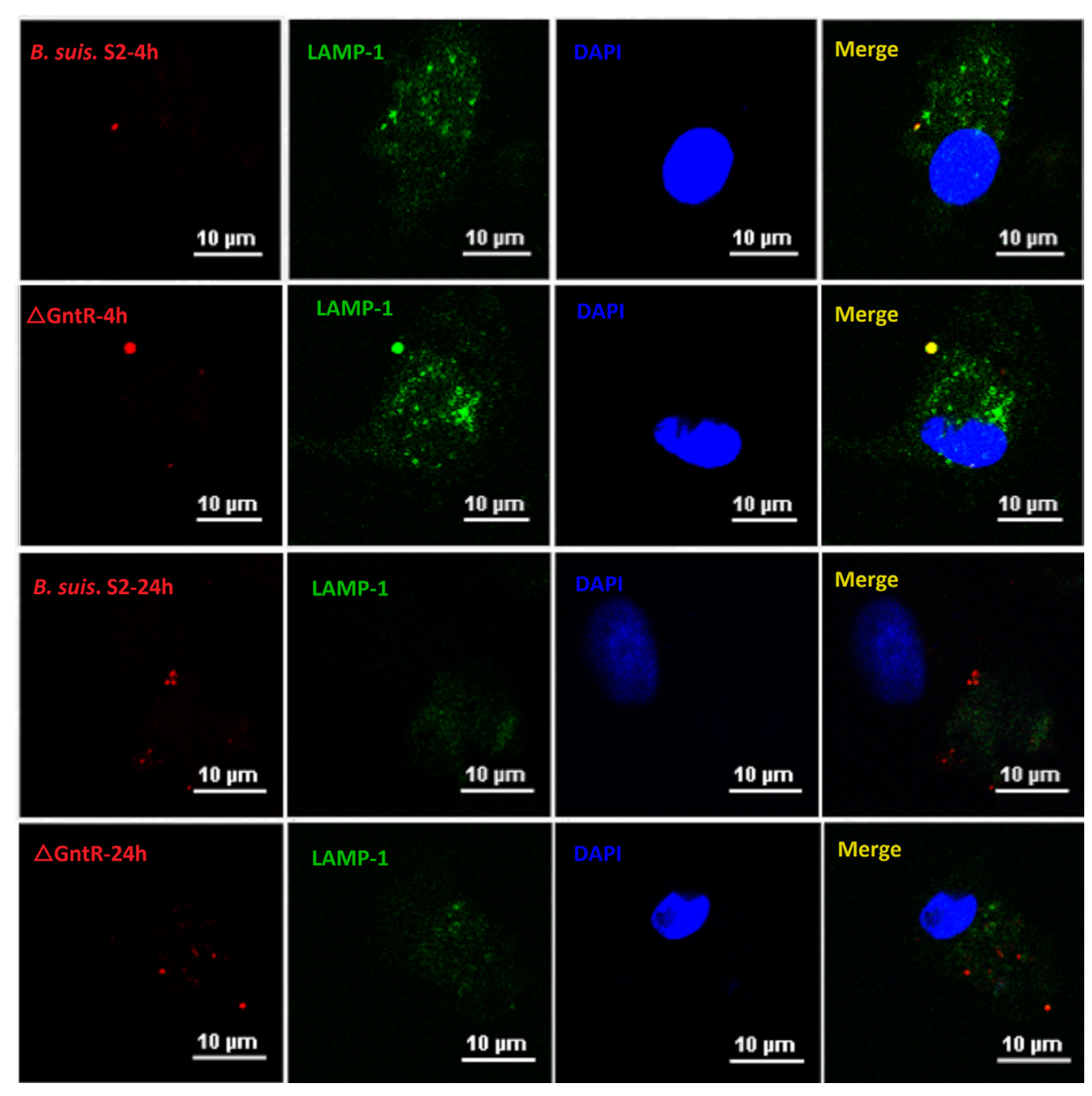

Figure 4: Intracellular survival and traffic of mutant strain $\Delta$ GntR in GAM cells. Representative confocal micrographs of GAMs infected with B. suis. S2 (red) and $\Delta \mathrm{GntR}$ (red) and immunostained for LAMP-1 (green) at 4 and $24 \mathrm{hr}$ post infection. The data shown are representative of 4-5 independent experiments. 
$\mathrm{h}$, and the PERK mRNA expression was even higher in the $\Delta$ GntR group than in the $B$. suis. S2 group at the same time point. However, 24 and $48 \mathrm{~h}$ after Brucella challenge, a significant decrease in PERK mRNA expression was observed in the $\Delta \mathrm{GntR}$ group compared with that in the B. suis. S2-infected and uninfected group (Figure 5F). Similar to the expression of PERK mRNA, ATF6 gene expression in the $\Delta \mathrm{GntR}$ mutant group was elevated at 0 $\mathrm{h}$ but decreased at 24 and $48 \mathrm{~h}$ compared with the $B$. suis. S2-infected or CONT group (Figure 5E).

\section{Changing ER stress affects $\Delta$ GntR intracellular growth in GAMs in vitro}

To more directly assess the role ER stress in Brucella replication in GAMs, the cells were treated with Tm (ER stress activator) and 4-PBA (ER stress antagonist) to induce or inhibit ER stress. As shown in Figure 6A and $6 \mathrm{~B}, 0.5 \mu \mathrm{g} / \mathrm{ml} \mathrm{Tm}$ and $1 \mu \mathrm{g} / \mathrm{ml}$ 4-PBA were used for the further experiments. Inducing ER stress with $0.5 \mu \mathrm{g} / \mathrm{ml}$ Tm significantly increased GRP78 protein expression and inhibited the proliferation of $B$. suis. S2 and $\Delta \mathrm{GntR}$ at $24 \mathrm{~h}$ post-infection compared with that in untreated infected GAMs (Figure 6C, 6D and 6E). Decreasing ER stress with $1 \mu \mathrm{g} / \mathrm{ml}$ 4-PBA significantly inhibited GRP78 protein expression and promoted B. suis. S2 and $\Delta \mathrm{GntR}$ proliferation at $24 \mathrm{~h}$ post-infection compared with untreated infected GAMs (Figure 6C, 6D and 6E). In addition, the proliferation of $\Delta \mathrm{GntR}$ was increased significantly compared with that of $B$. suis. S2 under ER stress inhibition (Figure 6E).

\section{$\Delta G n t R$ reduces the production of inflammatory cytokines in macrophages}

To determine the effects of GntR on the secretion of inflammatory cytokines by the infected cells, we collected the supernatant from GAMs infected with $B$. suis. S2 and $\Delta \mathrm{GntR}$ at 12, 24 and $48 \mathrm{~h}$, and measured the secretion levels of inflammatory cytokines IFN- $\gamma, \mathrm{TNF}-\alpha$, IL- $1 \beta$ and IL-10 by ELISA. The supernatant from GAMs infected with $\Delta$ GntR produced higher amounts of TNF- $\alpha$ than those from the B. suis. S2-infected and uninfected groups at $12 \mathrm{~h}$, and remained higher than those from the $B$. suis. S2-infected group at $24 \mathrm{~h}$ (Figure 7B). The supernatant concentration of IFN- $\gamma$ was elevated in the
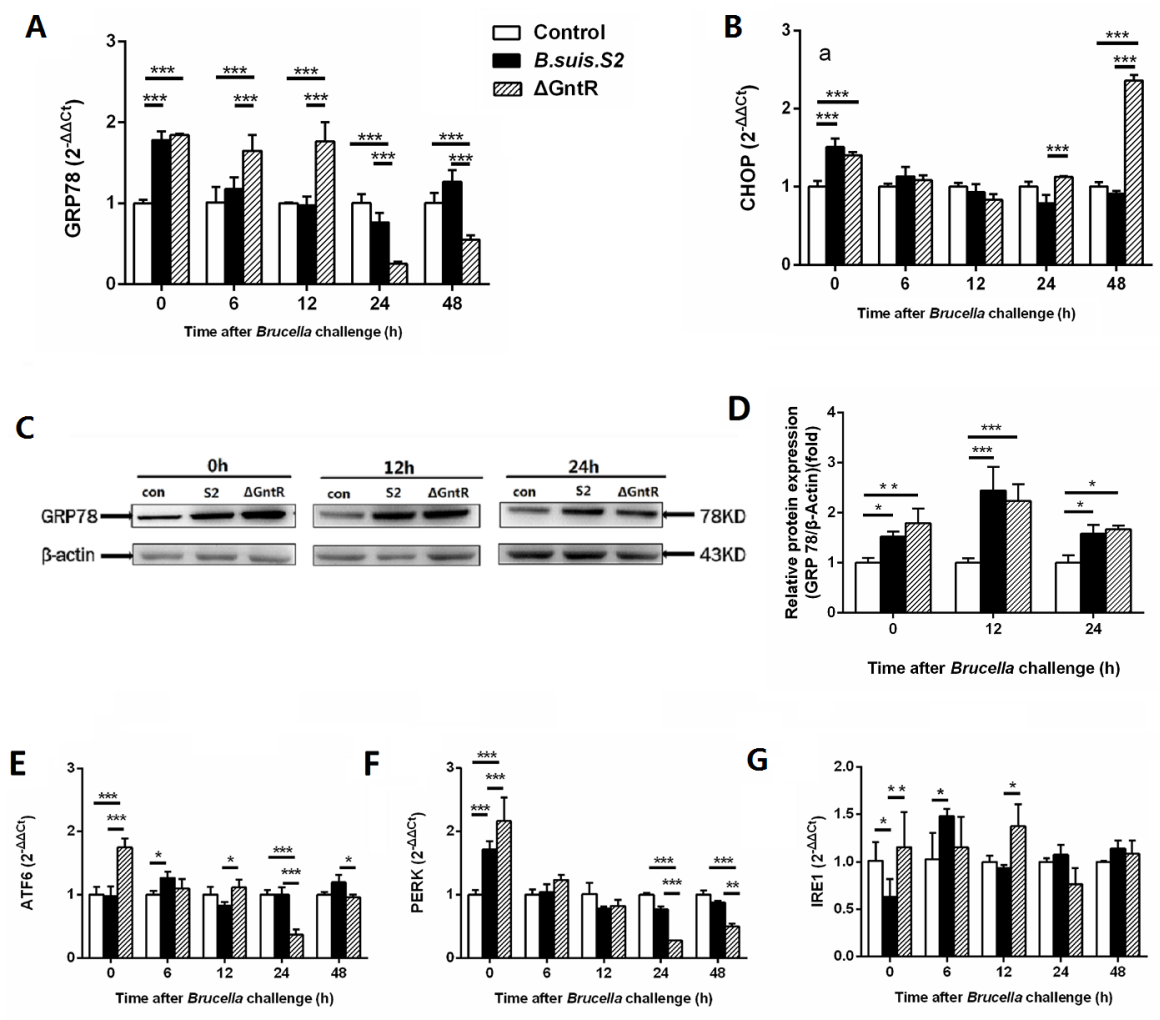

Figure 5: Comparison of ER stress in the B. suis. S2-infected and $\Delta$ GntR-infected strains in GAMs. The mRNA expression levels of GRP78 (A), CHOP (B) ATF6 (E), PERK (F), IRE1 (G) in GAMs was analyzed using quantitative real-time PCR. GAMs collected after $B$. suis. S2 or $\Delta \mathrm{GntR}(\mathrm{MOI}=100: 1)$ challenge at $0,6,12,24$ and $48 \mathrm{~h}$. Data are presented as the mean \pm standard error ${ }^{* *} P<0.01$, ${ }^{* * *} P<0.001$. (C) GAMs were infected with 100 MOI of B. suis. S2 or $\Delta$ GntR for 0,12 and $24 \mathrm{~h}$, followed by lysis and detection of GRP78 expression by Western blotting. (D) Quantification of band intensities was determined by densitometric analysis. The data shown are representative of 3 independent experiments. 
A

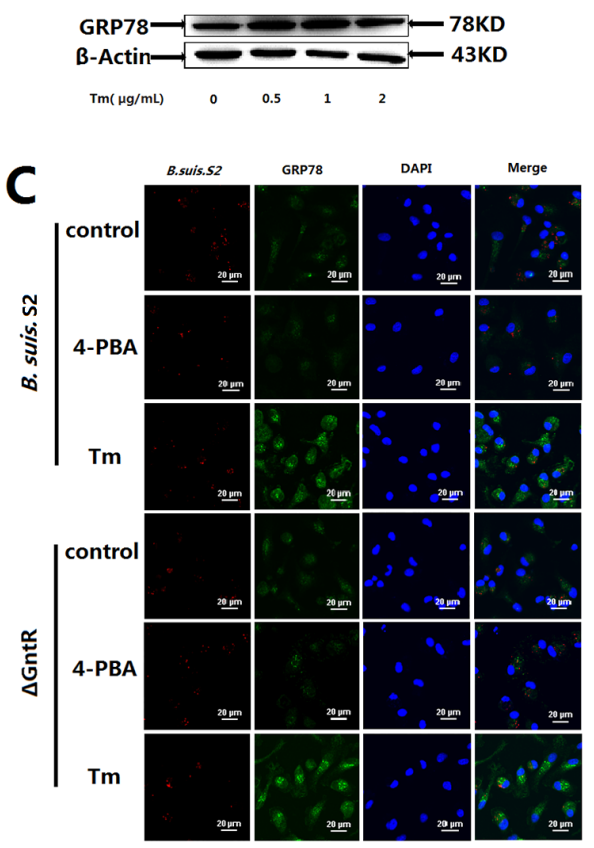

B

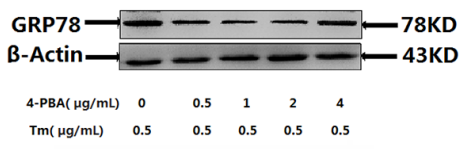

D

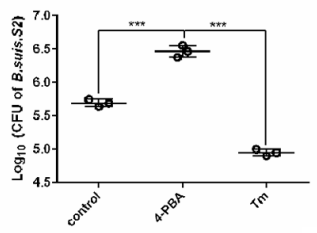

E

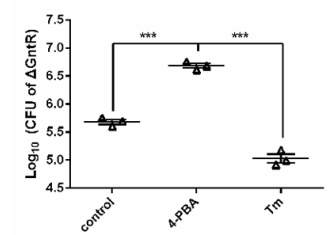

$\mathbf{F}$

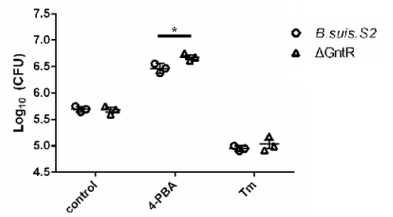

Figure 6: Changing ER stress influences the $B$. suis. S2 and $\Delta$ GntR intracellular survival. To establish ER stress activated and inhibited model, the UPR marker GRP78 in GAMs treated with different concentrations of (A) Tm or (B) 4-PBA was analyzed using Western-blot. The data shown are representative of 3 independent experiments. (C) Representative confocal micrographs of GRP78 protein (green) in GAMs infected with B. suis. S2 (red) or $\Delta \mathrm{GntR}$ (red) only or plus $0.5 \mu \mathrm{g} / \mathrm{mL}$ Tm or $1 \mu \mathrm{M}$ 4-PBA at $24 \mathrm{~h}$. The data shown are representative of 4-5 independent experiments. (D-F) GAMs were infected with $100 \mathrm{MOI} B$. suis. S2 (D) and $\Delta \mathrm{GntR}$ (E). Next, $0.5 \mu \mathrm{g} /$ $\mathrm{mL}$ Tm or $1 \mu \mathrm{M}$ 4-PBA was added before infection. Cells were lysed after $24 \mathrm{~h}$ of B. suis. S2 infection. CFUs were determined by transfer to dilution plates. CFU numbers are shown on a $\log _{10}$ scale. The data represent the means \pm standard error deviations from 3 independent experiments and were analyzed by one-way ANOVA. ${ }^{* * *} P<0.001$.
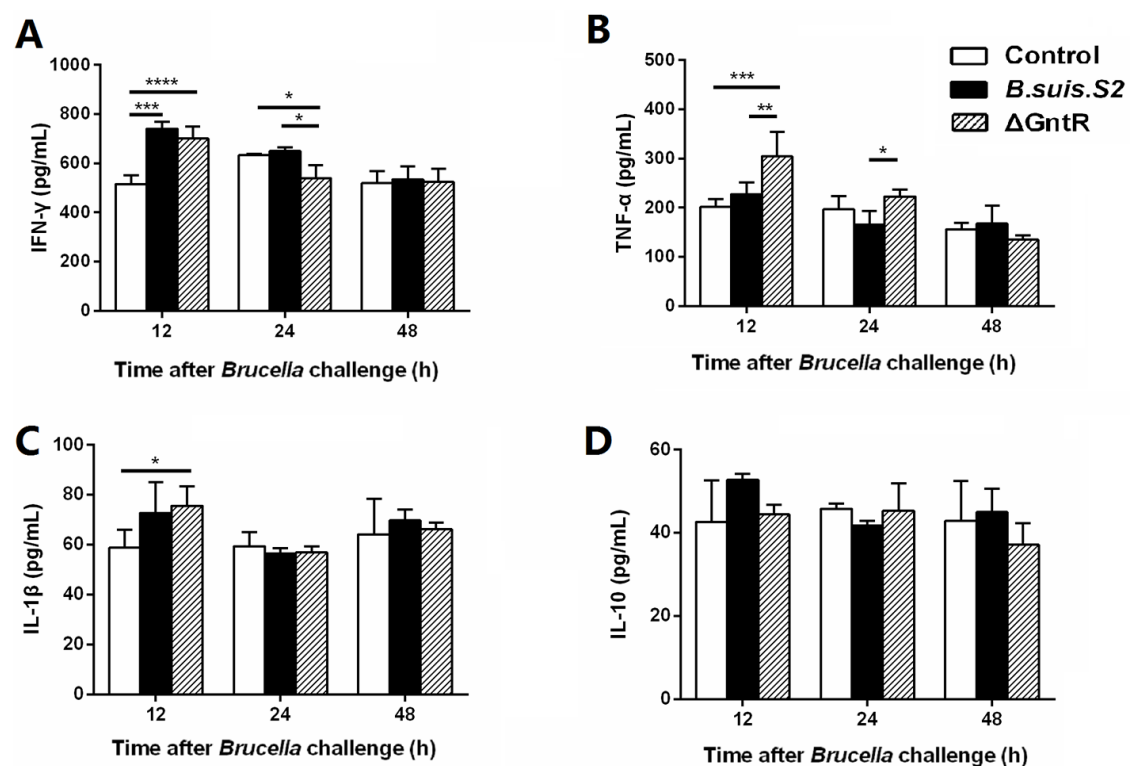

Figure 7: Production of inflammatory cytokines in the B. suis. S2- and $\Delta$ GntR-infected macrophages. GAMs were infected with B. suis. S2 and $\Delta \mathrm{GntR}$ at an MOI of 100 . At 12, 24 and $48 \mathrm{~h}$ post infection, supernatant samples were collected, and the IFN- $\gamma$ (A), TNF- $\alpha$ (B), IL-1 $\beta$ (C) and IL-10 (D) secretion levels were assayed by ELISA. The data represent the means \pm standard errors and were analyzed by two-way ANOVA. ${ }^{*} P<0.05,{ }^{* *} P \leq 0.01,{ }^{* * *} P \leq 0.001$. 
$\Delta$ GntR- and B. suis. S2-infected group compared with that in the uninfected group at $12 \mathrm{~h}$ after challenge. However, The IFN- $\gamma$ production level was lower in $\Delta \mathrm{GntR}$-infected GAMs than in B. suis. S2-infected and uninfected GAMs at $24 \mathrm{~h}$ (Figure 7A). Notably, at $12 \mathrm{~h}$ after challenge, the secretion levels of IL- $1 \beta$ were higher in the $\Delta$ GntR group than in the uninfected group (Figure 7C). No difference in the IL-10 production level was observed among the 3 groups (Figure 7D). These results indicate that $\Delta \mathrm{GntR}$ mutant could induce the secretion of inflammatory cytokines but not that of anti-inflammatory cytokines.

\section{DISCUSSION}

Brucellosis is one of the highly contagious zoonotic diseases worldwide with more than 500,000 new people infections annually. Airborne transmission has long been regarded as an important way to infect human and may be also involved in farm animal cases [22]. Alveolar macrophages (AM) is the first phagocytic cells contacted by inhaled of aerosols contaminated with Brucella. However, the interaction of B. suis. S2 with the goat AM has scarcely been studied. Here, we extrapolate that the $B$. suis. $\mathrm{S} 2$ could replicate and induce an unfolded protein response in goat AM.

Bacterial transcriptional regulators regulate various virulence genes at the transcription initiation step in response to cellular signals. The GntR family is one of the most abundant and widely distributed transcriptional regulators families. More than 8,500 GntR family members have been annotated in diverse bacterial genomes and several members of GntR family in Brucella have been proved to be involved in the virulence of the pathogen $[19,23]$. However, the function of the most predicted GntR members in B. suis. S2 is unknown. Our study found that predicted GntR transcription regulator BSS2_II0438 is associated with the expression of T4SS structural genes (VirB2, VirB6, VirB8), Which was consistent with previous studies $[19,24]$. VirBs are essential for Brucella intracellular replication, modulation of host immune functions, and establishment of chronic brucellosis $[1,25]$.

Upon phagocytosis, Brucella reside within Brucellacontaining vacuoles (BCVs), which undergo interactions with early endosomes and late endosomes in a controlled manner and partially fuse with lysosomes to become endosomal BCV (eBCVs). In this step, approximately $90 \%$ of Brucella are degraded, and the remaining $10 \%$ survive [26]. Previous studies have shown that B. abortus invades and replicates in murine AM, which did not induce cytotoxicity. AM could constitute a durable replicative niche for Brucella in infection [4]. Our results show that B. suis. S2 replicates in goat AM. deletion of BSS2 II0438 GntR of B. suis. S2 did not affect the intracellular survival stage of the bacteria's cycle. However, VirB2 mutant reduced the replication competence of Brucella in the $\mathbf{J 7 7 4}$ macrophages cell line [27]. This phenomenon partly because $\Delta \mathrm{GntR}$ could not efficiently evade fusion

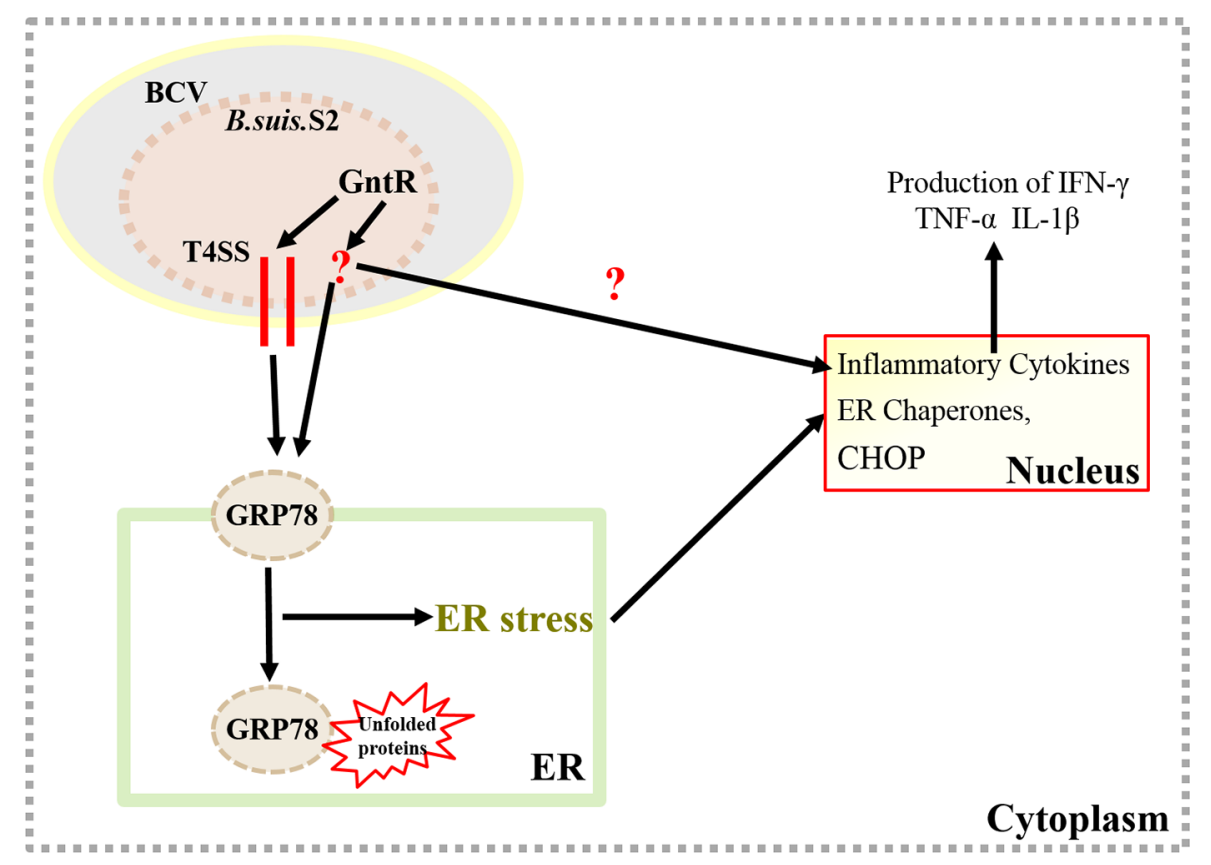

Figure 8: Update model of the GAMs response to B. suis. S2 infection. The GntR-like transcription regulator BSS2 II0438 can decrease the T4SS genes expression of B. suis. S2, which is involved in ERS pathway and the inflammatory response in GAMs. 
Table 1: Primers used in the study

\begin{tabular}{|c|c|c|c|}
\hline Primer & Accession no & Sequence & Product (bp) \\
\hline GntR-UF & СР006962.1 & CTGCAGAACCGCTTCAGCCATTCTTCCA & 607 \\
\hline GntR-UR & & TCTAGATATCGTTCAGATACATAAAGCTCGG & \\
\hline GntR-DF & СР006962.1 & GAGCTCAGCGGCCCATATCGG & 825 \\
\hline GntR-DR & & GAATTCATCGGGCTTTCCGCAGT & \\
\hline G-F & EF153731.1 & TCTAGATTGACATAAGCCTGTTCGGTTCGTA & 751 \\
\hline G-R & & GAGCTCTTAGGTGGCGGTACTTGGGTCGATA & \\
\hline Out-GntR-F & СР006962.1 & AGCATGGAACAGAACGTCATAATCA & \\
\hline Out-GntR-R & & TGCAAGGAAGGAACGGATT & \\
\hline RT-GntF & СР006962.1 & GCACTACAGGCAGGACACG & 280 \\
\hline RT-GntR & & TGAGGCTCAACCCGCTAA & \\
\hline RT-virB1-F & СР006962.1 & TGCCATTTCTTGTCCTCG & 143 \\
\hline RT-virB1-R & & AAGGGAAACGGGTTGACG & \\
\hline RT-virB2-F & СР006962.1 & CGCTGCAATCGAGCCTAA & 154 \\
\hline RT-virB2-R & & GTGCCGGAATGCCATCTT & \\
\hline RT-virB6-F & СР006962.1 & ATATCAGCACCTATTCGGAGTG & 154 \\
\hline RT-virB6-R & & ATGGCTGCGATGTTCCAC & \\
\hline RT-virB8-F & СР006962.1 & GACAAGCAATATGGCTCAAA & 193 \\
\hline RT-virB8-R & & CCGATTCCGACATCAAAGA & \\
\hline RT-virB9-F & СР006962.1 & ATTTACGCAAGGCTGGGAGT & 216 \\
\hline RT-virB9-R & & TGAACACGATAGGCAACA & \\
\hline RT-virB11-F & СР006962.1 & CCGCAAGCCGTCTTTCAC & 297 \\
\hline RT-virB11-R & & CGATCACCCTTTCAAGCTGTAC & \\
\hline RT-16sRNA-F & СР006962.1 & GTGGAATTCCGAGTGTAGAGG & 371 \\
\hline RT-16sRNA-R & & GTCCAGCCTAACTGAAGGATAG & \\
\hline RT-CHOP-F & AY943948.1 & AGGACCACCAGAGGTCACAC & 193 \\
\hline RT-CHOP-R & & TGCCACTTTCCTTTCGTTTT & \\
\hline RT-GRP78-F & DQ029323.1 & TGAAACTGTGGGAGGTGTCA & 171 \\
\hline RT-GRP78-R & & TCGAAAGTTCCCAGAAGGTG & \\
\hline RT-PERK-F & XM_005686691.1 & CCCCATCCGCTACTGAACG & 151 \\
\hline RT-PERK-R & & GGGCTGCTGGAGTGTCTTG & \\
\hline RT-IRE1-F & XM_005694366.1 & ACTCCCTCAACATCGTTCACAG & 208 \\
\hline RT-IRE1-R & & CTCCTTGCAGTCTTCGCTCA & \\
\hline RT-ATF6-F & AY942654 & AACCAGTCCTTGCTGTTGCT & 204 \\
\hline RT-ATF6-R & & CTTCTTCTTGCGGGACTGAC & \\
\hline RT-GAPDH-F & XM_005680968.1 & GGCGCCAAGAGGGTCAT & 100 \\
\hline RT-GAPDH-R & & GTGGTTCACGCCCATCACA & \\
\hline
\end{tabular}


with lysosomes in GAMs. Studies have shown that AM differ from other monocytic/macrophagic populations in the intracellular replication kinetics for certain pathogens $[11,12]$ These results deduce that the BSS2_II043 GntR protein is not evolved in the induction of eBCV by $B$. suis. S2 or that it may work redundantly with other, asyet unidentified, effectors to elicit this stage. eBCVs are converted into replication-permissive organelles (rBCVs) via the accretion of ER-derived membranes and exclusion of endosomal membranes. rBCVs can interact with the ER and promote Brucella replication. Brucella fuses with the endoplasmic reticulum (ER) and provokes an ER stress response called the unfolded protein response. The unfolded protein response (UPR) induced by viruses and bacteria is crucial for their colonization, replication and persistent infection in infected cells. GRP78, as a monitor of ER stress, constrains the biology and life cycles of Brucella. Our previous study showed that enhancing the expression of GRP78 promoted the proliferation of $B$. suis. S2, and inhibiting GRP78 protein expression greatly diminished the numbers of B. suis. S2 in GTC lines. In addition, altered ER Stress with 4-PBA or Tm affects the intracellular growth of B. suis. S2 in GTCs [17]. This finding is in line with our results that enhancing GRP78 protein expression with Tm inhibited the proliferation of Brucella in GAMs. Moreover, inhibiting GRP78 protein expression with 4-PBA increased the number of Brucella CFUs in GAMs. B. abortus exploits VceC to interact with GRP78 and localizes to the ER in HeLa cells [27]. The apparent effect of TUDCA in Brucella inducing GRP78 expression is caused by the greatly diminished numbers of bacteria [28]. Increasing GRP78 expression promotes the proliferation of $B$. suis. S2 in GTCs by inhibiting the apoptosis of B. suis. S2-infected GTCs [17]. Notably, our results showed that $\Delta \mathrm{GntR}$ increased greatly the number of Brucella CFUs in the inactive ER stress state compared with B. suis. S2 in GAMs. These results imply that GntR is potentially involved in ER stress and triggers the UPR via the T4SS of B. suis. S2 or other ways.

Inflammatory response is beneficial for host to protection against bacteria invasion [29]. In the early stages of brucellosis, Brucella change the cytokine level of IFN- $\gamma$, TNF- $\alpha$, and IL-10 [30]. Recent study clarify that Brucella have capability to inhibit the secretion of IFN- $\gamma$ and increase the secretion of IL-10 that enhances chronic infection [31]. In the present study, we found that goat AMs respond to Brucella infection with an increased secretion of IFN- $\gamma$ in the early stages of infection but no effect on secretion of TNF- $\alpha$, IL- $1 \beta$ and IL-10. IFN$\gamma$-mediated type I immune responses are essential for clearance of Brucella [32]. The significant production of IFN- $\gamma$ by Brucella infected goat AMs deduces that GAMs contribute to the clearance of Brucella during aerosols infection. Interestingly, we found that $\Delta \mathrm{GntR}$ infected GAMs resulted in increased secretion of TNF- $\alpha$, IL-1 $\beta$ and IFN- $\gamma$. Previous study has shown that mouse alveolar macrophages response to Brucella infection with a marked increase in secretion of TNF- $\alpha$ and IL$1 \beta$ [4]. TNF- $\alpha$ controls macrophage activation and neutrophils recruitment to the infected site. the expression of TNF- $\alpha$ enhances the elimination effect on Brucella by reinforcement bactericidal and antigen-presenting functions of macrophages [33, 34]. The significant production of TNF- $\alpha$ by $\Delta$ GntR-infected GAMs suggests that BSS2_II043 GntR protein is essential for Brucella to evade the host elimination. Consistent with previous study, Brucella-infected murine AMs resulted in increased expression of IL-1 $\beta$, but the effect of IL-1 $\beta$ for the response to inhaled Brucella had not been explored [4]. The anti-inflammatory cytokine, IL-10 was not elicited by both Brucella and $\Delta \mathrm{GntR}$ infection. Study indicated that lack of production of IL-10 resulted in an increased ability of mice to control B. abortus infection. The UPR has increasingly been shown to associated with inflammatory response. Activation of IRE1 by ER stress can activate the mitogen-activated protein kinase (MAPK), JNK, which is a key player in the response to inflammatory stimuli [35]. Macrophages undergoing ER stress induction enhanced IL-1 $\beta$, IL- 6 and TNF- $\alpha$ production in response to LPS stimulation in vitro [36]. In Brucella-infected macrophages, VceC can activate IRE1-dependent secretion of IL-6 and TNF [27]. Although residing in the ER might promote the survival of Brucella, it is possible that UPR induction might function as an innate immune mechanism against invading bacteria. Take these results extrapolated that BSS2_II043 GntR protein is essential for B. suis. S2 survival in goat AMs. Further studies are needed to clarify the mechanisms of the BSS2_II043 GntR protein involved in regulating the inflammatory response in vivo.

In this study, our results provide novel evidence that the GntR-like transcription regulator BSS2_II0438 can decrease the T4SS genes expression of B. suis. S2, which is involved in ERS pathway and the inflammatory response of GAMs (Figure 8). Our results may provide new insights into understanding the interaction between rBCV and ER during Brucella infections. The findings will also provide new targets to prevent and control Brucella infections.

\section{MATERIALS AND METHODS}

\section{Ethics statement}

The sampling procedures were compliance with the "Guidelines on Ethical Treatment of Experimental Animals" (2006) No. 398 established by the Ministry of Science and Technology, China.

All animals were treated in strict accordance with the "Guidelines on Ethical Treatment of Experimental Animals" (2006) No. 398 from the Ministry of Science and Technology, China. The sampling procedures in the present study had received prior approval from the 
Experimental Animal Manage Committee of Northwest A\&F Universit with the approval license number was $2017 Z X 08008005$.

\section{Bacterial strains and cells}

B. suis. S2 cells were obtained from the Chinese Veterinary Culture Collection Center (Beijing, China). B. suis. S2 was cultured in tryptic soy broth (TSB) or tryptic soy ager (TSA, Takana). The number of B. suis. S2 cells was counted by plating on TSA. This Escherichia coli strain DH5a (Takana) was grown on Luria-Bertani medium. When appropriate, $50 \mu \mathrm{g} / \mathrm{mL}$ ampicillin, kanamycin and gentamicin were respectively added. Plasmid PUC19 was purchased from Takana.

Goat alveolar macrophages (GAMs) were collected by bronchial lavage from the lungs of healthy 5 -month-old Guanzhong dairy goats. Donor animals were determined to be Brucella free. GAMs were cultured in RPMI-1640 medium (HyClone) supplemented with $15 \%$ fetal bovine serum (FBS, Gibco) at $37^{\circ} \mathrm{C}$ with $5 \% \mathrm{CO}_{2}$.

\section{Construction of the mutant strain $\Delta G$ GntR}

$\Delta \mathrm{GntR}$ was constructed as described previously [37]. Briefly, primers for construction were designed using the sequence GntR in the B. suis. S2 genome and PBBR1MCS-5. The 607-bp upstream fragment, 825-bp downstream fragment and 751-bp gentamicin fragment were obtained in three independent PCR reactions using primer STAR Max Mix with primer pairs GntR-UF/GntRUR, GntR-DF/GntR-DR and G-F/G-R. After purification by gel extraction, the three fragments were cloned into PMD19T-simple and then were digested with Pst 1 and $X b a \mathrm{I}, S a c \mathrm{I}$ and $E c o R \mathrm{I}, X b a \mathrm{I}$ and $S a c \mathrm{I}$ sequentially and then subcloned into the $X b a \mathrm{I}-$ and SacI-digested PUC19 plasmid. The recombinant plasmid with the correct sequence was designated PUC19-GntR and was introduced into DH5 $\alpha$. B. suis. S2 was electroporated with PUC19-GntR. The potential GntR deletion mutant $\Delta$ GntR was selected by plating on TSA-containing gentamicin, which was then verified by PCR, quantitative real-time PCR (qRT-PCR) and Western blotting. Primers were designed according to the strain $B$. suis. S2 genome in Table 1.

\section{Cell infection assay}

Cell infection was performed as previously reported [17]. Briefly, GAMs were seeded in six-well plates $(2 \times$ $10^{6}$ cells per well) and were infected with $B$. suis. S2 and $\triangle$ GntR at 100:1 MOI. After $4 \mathrm{~h}$ of incubation, the GAMs were washed three times with PBS and then further cultured with cell culture medium containing $50 \mu \mathrm{g} / \mathrm{mL}$ kanamycin to eliminate $B$. suis. S2 and $\Delta \mathrm{GntR}$ adhering to the GAMs and in the culture medium. After $1 \mathrm{~h}$, the GAMs were washed three times with PBS and were further cultured with cell culture medium containing $25 \mu \mathrm{g} / \mathrm{mL}$ kanamycin to avert continuous infection. This time was considered the $0 \mathrm{~h}$ and time point of treatment with Tm (ER stress activator) and 4-PBA (ER stress antagonist). The cells were collected, and relevant experiments were performed at specific times $(0,6,12,24$, and $48 \mathrm{~h})$.

For intracellular survival assays, wells of infected cells at $0,6,12,24$ and $48 \mathrm{~h}$ were washed and incubated with $500 \mu \mathrm{L}$ of $0.5 \%$ Triton X-100 in PBS for $10 \mathrm{~min}$. The lysates were diluted in PBS and were plated onto TSA at $37^{\circ} \mathrm{C}$ with $5 \% \mathrm{CO}_{2}$ for $72 \mathrm{~h}$. The number of bacteria at each time point were determined.

For adherence, wells of infected cells at $0 \mathrm{~h}$ were washed and incubated with $500 \mu 1$ of $0.5 \%$ Triton X-100 in PBS for $10 \mathrm{~min}$. Next, the cells were incubated with $500 \mu \mathrm{L}$ of $0.5 \%$ Triton X-100 in PBS for $10 \mathrm{~min}$. The lysates were diluted in PBS and were plated onto TSA to determine the colony-forming units.

\section{RNA isolation and quantitative real time PCR}

Strains $B$. suis. S2 and $\Delta \mathrm{GntR}$ were collected at the exponential phase. The GAMs of infected B. suis. S2 and $\Delta$ GntR were collected at $0,6,12,24$ and $48 \mathrm{~h}$. Total RNA was extracted using TRIzol (Invitrogen, Inc., Carlsbad, CA, USA). RNA was subjected to reverse transcription using the Vazyme RT Reagent Kit according to the manufacturer's protocols. Next, qRT-PCR was performed using an ABI 7500 Sequencing Detection System and SYBR Premix Ex Taq ${ }^{\mathrm{TM}}$. Primers were designed according to the strain B. suis. S2 genome in Table 1. The relative transcription levels were calculated using the $2^{-}$ $\Delta \Delta C \mathrm{Ct}$ method.

\section{Western-blotting analysis}

B. suis. S2-infected GAMs were harvested in a tube and then were lysed on ice for 30-45 min in lysis buffer. The supernatant was obtained by centrifugation for $15 \mathrm{~min}$ at $14,000 \mathrm{rpm}$ at $4^{\circ} \mathrm{C}$. The protein concentration was determined by the BCA assay. Total cellular protein was extracted with $5 \times$ SDS-PAGE loading buffer after boiling for $5 \mathrm{~min}$ in water. Samples were electrophoresed on a $12 \%$ polyacrylamide gel for SDS-PAGE. The gels were then electro-transferred onto PVDF membranes. The membranes were blocked for $1 \mathrm{~h}$ in Tris-buffered saline containing $0.5 \%$ Tween-20 (TBST) with 5-10\% skimmed milk at room temperature, and then were incubated overnight at $4^{\circ} \mathrm{C}$ in blocking solution containing rabbit anti mouse GRP78 antibody (Proteintech, 11587-1-AP, 1:1000 dilution) or mouse anti-human $\beta$-actin (Proteintech, 60008-1-lg, 1:2000 dilution). The membranes were washed five times with TBST for $5 \mathrm{~min}$ and then were incubated for $1 \mathrm{~h}$ with the goat anti rabbit $\operatorname{IgG}$ or goat anti mouse IgG antibody conjugated to HRP (Invitrogen, $31460,31430,1: 5000$ dilution). Finally, the membranes 
were washed five times in TBST for $5 \mathrm{~min}$. The blots were visualized using the Gel Image System (Tannon, Biotech, Shanghai, China).

\section{Immunofluorescence assay}

GAMs were seed on $15-\mathrm{mm}$ glass diameter coverslips in 24-well plates and were infected with B. suis. $\mathrm{S} 2$ and $\Delta \mathrm{GntR}$ at $100 \mathrm{MOI}$. Immunofluorescence staining of LAMP-1 and GRP78 in B. suis. S2 was performed. At 0 and $24 \mathrm{~h}$ pi, infected cells were washed twice with PBS and then were fixed with $4 \%$ paraformaldehyde at room temperature for $30 \mathrm{~min}$. After three washes with PBS, cells were incubated with PBS containing $0.25 \%$ Triton $\mathrm{X}-100$ at room temperature for $20 \mathrm{~min}$. After three washes with PBS, goat anti-brucella polyclonal antibody (1:100 dilution), rabbit anti- mouse GRP78 monoclonal antibody (Proteintech, 11587-1-AP, 1:200 dilution) and mouse antiLAMP-1 monoclonal antibody (Biolegend, 121601, 1:200 dilution) were used as the primary antibody. Donkey antigoat alexa fluor 555, Donkey anti-mouse alexa fluor 488 (Life technologies, 1736967) and Donkey anti-rabbit alexa fluor 488 (Life technologies, 1796365) were used as the secondary antibody at 1:200 dilutions. Next, coverslips were mounted on glass slides, and cells were observed under a microscope. Assays were performed in triplicate.

\section{ELISA}

GAMs cultured in 6-well plates were infected with B. suis. S2 or $\Delta \mathrm{GntR}$ at an MOI of 100:1 in triplicate wells. At 12, 24 and $48 \mathrm{~h}$ post infection, IL-1 $\beta$ (Biomatik, EKC32210), IL-10 (Biomatik, EKU05174), IFN- $\gamma$ (Novetinbio, BG-GT11412) and TNF- $\alpha$ (Biomatik, EKU07943) production in the culture supernatants was tested using an ELISA kit according to the manufacturer's instructions.

\section{Statistical analysis}

Statistical analysis was performed using Graphpad Prism software 6 (GraphPad software Inc., La Jolla, CA, USA). Statistical significance was determined using twoway ANOVA or one-way ANOVA. $P$ values less than 0.05 were considered statistically significant.

\section{Abberviations}

AM, Alveolar macrophages; ER, endoplasmic reticulum; B. suis. S2, Brucella suis vaccine strain 2; T4SS, type IV secretion system; GAMs, goat alveolar macrophages; IFN- $\gamma$, interferon- $\gamma$; IL- $1 \beta$, interleukin- $1 \beta$; tumor necrosis factor- $\alpha$, TNF- $\alpha$; BCV, Brucella containing vacuole; UPR, unfolded protein response; IRE1, inositolrequiring kinase 1; PERK, pancreatic ER eIF2a kinase; ATF6, activating transcription factor 6; CHOP, C/EBPhomologous protein; 4-PBA, 4 Phenyl butyric acid.

\section{Author contributions}

AW, YJ, and DZ designed the study and experiments. FZ, DZ, MQ, GZ, FB, JL, and HL performed the research and analysed data. HC, PL, KT and WL analysed data. All authors interpreted and discussed the data. DZ, FZ, PJ and AW wrote the manuscript. All authors read and approved the final manuscript.

\section{ACKNOWLEDGMENTS}

The authors would like to thank all members of our groups.

\section{CONFLICTS OF INTEREST}

The authors declare no potential conflicts of interest.

\section{GRANT SUPPORT}

This study was supported by grant from the National Natural Science Foundation of China 31672584; the Sci-tech Innovation Project of Shaanxi Province 2016TZC-N-13-5; China Postdoctoral Science Foundation 2016M602883. Natural Science Basic Research Plan in Shaanxi Province of China 2017JQ3010. National Training Program of Innovation and Entrepreneurship for Undergraduates 201610712022.

\section{REFERENCES}

1. Byndloss MX, Tsolis RM. Brucella spp. virulence factors and immunity. Annu Rev Anim Biosci. 2016; 4:111-27.

2. von Bargen K, Gorvel JP, Salcedo SP. Internal affairs: investigating the Brucella intracellular lifestyle. FEMS Microbiol Rev. 2012; 36:533-62.

3. Pappas G, Papadimitriou P, Akritidis N, Christou L, Tsianos EV. The new global map of human brucellosis. Lancet Infect Dis. 2006; 6:91-9.

4. Ferrero MC, Hielpos MS, Carvalho NB, Barrionuevo P, Corsetti PP, Giambartolomei GH, Oliveira SC, Baldi PC. Key role of Toll-like receptor 2 in the inflammatory response and major histocompatibility complex class ii downregulation in Brucella abortus-infected alveolar macrophages. Infect Immun. 2014; 82:626-39.

5. de Figueiredo P, Ficht TA, Rice-Ficht A, Rossetti CA, Adams LG. Pathogenesis and immunobiology of brucellosis review of Brucella-host interactions. Am J Pathol. 2015; 185:1505-17.

6. De Bolle X, Crosson S, Matroule JY, Letesson JJ. Brucella abortus cell cycle and infection are coordinated. Trends Microbiol. 2015; 23:812-21.

7. Van der Henst C, de Barsy M, Zorreguieta A, Letesson JJ, De Bolle X. The Brucella pathogens are polarized bacteria. Microbes Infect. 2013; 15:998-1004. 
8. Zhu L, Feng Y, Zhang G, Jiang H, Zhang Z, Wang N, Ding J, Suo X. Brucella suis strain 2 vaccine is safe and protective against heterologous Brucella spp. infections. Vaccine. 2016; 34:395-400.

9. Surewicz K, Aung H, Kanost RA, Jones L, Hejal R, Toossi Z. The differential interaction of p38 MAP kinase and tumor necrosis factor-alpha in human alveolar macrophages and monocytes induced by Mycobacterium tuberculois. Cell Immunol. 2004; 228:34-41.

10. Kapetanovic R, Parlato M, Fitting C, Quesniaux V, Cavaillon JM, Adib-Conquy M. Mechanisms of TNF induction by heat-killed Staphylococcus aureus differ upon the origin of mononuclear phagocytes. Am J Physiol Cell Physiol. 2011; 300:C850-9.

11. van Riel D, Leijten LM, van der Eerden M, Hoogsteden HC, Boven LA, Lambrecht BN, Osterhaus AD, Kuiken T. Highly pathogenic avian influenza virus H5N1 infects alveolar macrophages without virus production or excessive TNF-alpha induction. PLoS Pathog. 2011; 7:e1002099.

12. Yu WC, Chan RW, Wang J, Travanty EA, Nicholls JM, Peiris JS, Mason RJ, Chan MC. Viral replication and innate host responses in primary human alveolar epithelial cells and alveolar macrophages infected with influenza H5N1 and H1N1 viruses. J Virol. 2011; 85:6844-55.

13. Salez L, Singer M, Balloy V, Creminon C, Chignard M. Lack of IL-10 synthesis by murine alveolar macrophages upon lipopolysaccharide exposure. Comparison with peritoneal macrophages. J Leukoc Biol. 2000; 67:545-52.

14. Starr T, Child R, Wehrly TD, Hansen B, Hwang S, LopezOtin C, Virgin HW, Celli J. Selective subversion of autophagy complexes facilitates completion of the Brucella intracellular cycle. Cell Host Microbe. 2012; 11:33-45.

15. Hetz C, Chevet E, Oakes SA. Proteostasis control by the unfolded protein response. Nat Cell Biol. 2015; 17:829-38.

16. Celli J, Tsolis RM. Bacteria, the endoplasmic reticulum and the unfolded protein response: friends or foes? Nat Rev Microbiol. 2015; 13:71-82.

17. Wang XG, Lin PF, Li Y, Xiang CX, Yin YL, Chen Z, $\mathrm{Du}$ Y, Zhou D, Jin YP, Wang AH. Brucella suis vaccine strain 2 induces endoplasmic reticulum stress that affects intracellular replication in goat trophoblast cells in vitro. Front Cell Infect Microbiol. 2016; 6:19.

18. Smith JA, Khan M, Magnani DD, Harms JS, Durward M, Radhakrishnan GK, Liu YP, Splitter GA. Brucella induces an unfolded protein response via TcpB that supports intracellular replication in macrophages. PLoS Pathog. 2013; 9:e1003785.

19. Haine V, Sinon A, Van Steen F, Rousseau S, Dozot M, Lestrate P, Lambert C, Letesson JJ, De Bolle X. Systematic targeted mutagenesis of Brucella melitensis $16 \mathrm{M}$ reveals a major role for GntR regulators in the control of virulence. Infect Immun. 2005; 73:5578-86.

20. Li Z, Wang S, Zhang H, Zhang J, Xi L, Zhang J, Chen C. Transcriptional regulator GntR of Brucella abortus regulates cytotoxicity, induces the secretion of inflammatory cytokines and affects expression of the type IV secretion system and quorum sensing system in macrophages. World J Microbiol Biotechnol. 2017; 33:60.

21. Wang Y, Ke Y, Xu J, Wang L, Wang T, Liang H, Zhang W, Gong C, Yuan J, Zhuang Y, An C, Lei S, Du X, et al. Identification of a novel small non-coding RNA modulating the intracellular survival of Brucella melitensis. Front Microbiol. 2015; 6:164.

22. Wallach JC, Samartino LE, Efron A, Baldi PC. Human infection by Brucella melitensis: an outbreak attributed to contact with infected goats. FEMS Immunol Med Microbiol. 1997; 19:315-21.

23. Hoskisson PA, Rigali S. Chapter 1: variation in form and function the helix-turn-helix regulators of the GntR superfamily. Adv Appl Microbiol. 2009; 69:1-22.

24. Xu YY, Wang SC, Li DJ, Du MR. Co-signaling molecules in maternal-fetal immunity. Trends Mol Med. 2017; 23:46-58.

25. Roux CM, Rolan HG, Santos RL, Beremand PD, Thomas TL, Adams LG, Tsolis RM. Brucella requires a functional Type IV secretion system to elicit innate immune responses in mice. Cell Microbiol. 2007; 9:1851-69.

26. Ke Y, Wang Y, Li W, Chen Z. Type IV secretion system of Brucella spp. and its effectors. Front Cell Infect Microbiol. 2015; 5:72.

27. de Jong MF, Starr T, Winter MG, den Hartigh AB, Child R, Knodler LA, van Dijl JM, Celli J, Tsolis RM. Sensing of bacterial type IV secretion via the unfolded protein response. MBio. 2013; 4:e0418-12.

28. Smith JA, Khan M, Magnani DD, Harms JS, Durward M, Radhakrishnan GK, Liu YP, Splitter GA. Brucella induces an unfolded protein response via TcpB that supports intracellular replication in macrophages. PLoS Pathog. 2013; 9:e1003785.

29. Medzhitov R. Origin and physiological roles of inflammation. Nature. 2008; 454:428-35.

30. Ahmed W, Zheng K, Liu ZF. Establishment of chronic infection: Brucella's stealth strategy. Front Cell Infect Microbiol. 2016; 6:30.

31. Wang Y, Chen Z, Qiu Y, Ke Y, Xu J, Yuan X, Li X, Fu S, Cui M, Xie Y, Du X, Wang Z, Huang L. Identification of Brucella abortus virulence proteins that modulate the host immune response. Bioengineered. 2012; 3:303-5.

32. Goenka R, Parent MA, Elzer PH, Baldwin CL. B celldeficient mice display markedly enhanced resistance to the intracellular bacterium Brucella abortus. J Infect Dis. 2011; 203:1136-46.

33. Zhang K, Wang H, Guo F, Yuan L, Zhang W, Wang Y, Chen C. OMP31 of Brucella melitensis 16M impairs the apoptosis of macrophages triggered by TNF-alpha. Exp Ther Med. 2016; 12:2783-9.

34. Gentilini MV, Velasquez LN, Barrionuevo P, Benitez PC, Giambartolomei GH, Delpino MV. Adrenal steroids modulate the immune response during Brucella abortus 
infection by a mechanism that depends on the regulation of cytokine production. Infect Immun. 2015; 83:1973-82.

35. Urano F, Wang X, Bertolotti A, Zhang Y, Chung P, Harding HP, Ron D. Coupling of stress in the ER to activation of JNK protein kinases by transmembrane protein kinase IRE1. Science. 2000; 287:664-6.

36. Shenderov K, Riteau N, Yip R, Mayer-Barber KD, Oland S, Hieny S, Fitzgerald P, Oberst A, Dillon CP, Green DR,
Cerundolo V, Sher A. Cutting edge: endoplasmic reticulum stress licenses macrophages to produce mature IL-1beta in response to TLR4 stimulation through a caspase-8- and TRIF-dependent pathway. J Immunol. 2014; 192:2029-33.

37. Gao JP, Tian MX, Bao YQ, Li P, Liu JM, Ding C, Wang SH, Li T, Yu SQ. Pyruvate kinase is necessary for Brucella abortus full virulence in BALB/c mouse. Vet Res. 2016; 47:87. 